\title{
Critérios para seleção do intervalo RR no eletrocardiograma para quantificação da arritmia sinusal respiratória
}

\author{
Renata Rodrigues Teixeira de Castro, Sergio Henrique Rodolpho Ramalho \\ e Antonio Claudio Lucas da Nóbrega \\ Departamento de Fisiologia, Instituto Biomédico, Universidade Federal Fluminense, Niterói, RJ
}

\section{RESUMO}

O sistema nervoso autônomo (SNA) sofre importantes adaptações ao treinamento físico aeróbico e alterações na sua função estão relacionadas à gênese e prognóstico de várias doenças. A variação da freqüência cardíaca em função da respiração constitui fenômeno natural no qual se baseia o teste da arritmia sinusal respiratória (ASR), utilizado para avaliar o funcionamento do ramo parassimpático do SNA sobre o sistema cardiovascular. Calcula-se a ASR através da razão entre o maior intervalo R-R do eletrocardiograma (ECG) durante a expiração (E) e o menor R-R na inspiração (I). Não existe, na literatura, padronização para escolha destes intervalos quando o ECG é registrado durante dois ciclos respiratórios consecutivos. O objetivo deste trabalho é comparar os métodos de escolha desses intervalos, indicando qual o critério que permite melhor avaliação da magnitude da ASR. Cinqüenta e cinco voluntários jovens foram submetidos a dois testes de ASR, sendo cada teste composto por dois ciclos respiratórios consecutivos. Foram então comparados os dois maiores índices E/I obtidos das seguintes maneiras: 1) E e I retirados do mesmo ciclo respiratório (intraciclo); 2) E e I retirados de qualquer um dos ciclos respiratórios (ciclo independente). Os índices E/I ciclo-independentes $\left(1^{\circ}\right.$ teste $=1,49 \pm$ 0,$03 ; 2^{\circ}$ teste $\left.=1,44 \pm 0,03\right)$ foram maiores do que os índices E/I obtidos intraciclo $\left[1^{\circ}\right.$ teste $=1,44 \pm 0,03 ; 2^{\circ}$ teste $=$ $1,41 \pm 0,03(\mathrm{P}<0,001)]$. Os índices $\mathrm{E} / \mathrm{I}$ ciclo-independentes do $1^{\circ}$ teste foram maiores do que os do $2^{\circ}$ teste $(\mathrm{P}=$ 0,04). A utilização de intervalos E e I escolhidos de forma independente do ciclo respiratório onde ocorreram permitiu observação da máxima variação da atividade parassim-

Endereço para correspondência:

Antonio Claudio Lucas da Nóbrega

Departamento de Fisiologia

Instituto Biomédico

Universidade Federal Fluminense

Rua Hernani Melo, 101

24210-130 - Niterói, RJ pática, mostrando a máxima magnitude da ASR. Em adendo, os resultados sugerem uma acomodação do reflexo no $2^{\circ}$ teste, tornando desnecessária a realização deste quando o $1^{\circ}$ prosseguir sem intercorrências técnicas.

Palavras-chave: Sistema nervoso autônomo. Arritmia sinusal respiratória. Testes autonômicos cardiovasculares.

\section{ABSTRACT \\ Selection of RR interval in the electrocardiogram to de- termine the respiratory sinus arrhythmia}

The autonomic nervous system (ANS) undergoes important adaptations to aerobic physical training and alterations in its function are related to the genesis and prognosis of various diseases. The heart rate fluctuation in response to respiration is a natural phenomenon in which is based the respiratory sinus arrhythmia test $(R S A)$, utilized to evaluate the function of the parasympathetic branch of the ANS in regulating the cardiovascular system. The RSA is calculated by the coefficient between the longer $R-R$ interval from the electrocardiogram (ECG) during the expiration (E) and the shorter $R-R$ interval during inspiration. So far, there is no standard way to select the $R-R$ intervals when the ECG is registered during two consecutive respiratory cycles. The purpose of this study was to compare the methods used to select these intervals, determining the most effective way to quantify the RSA. Fifty-five young volunteers performed two consecutive RSA tests, each one composed by two consecutive respiratory cycles. The two largest $E / I$ indexes calculated by either one of these two ways were then compared: 1) both $E$ and I derived from the same respiratory cycle (intra-cycle); 2) $E$ and I from any one of the two respiratory cycles (cycle-independent). The cycleindependent $E / I$ indexes $\left(1^{\text {st }}\right.$ test $=1.49 \pm 0.03 ; 2^{\text {nd }}$ test $=$ $1.44 \pm 0.03)$ were higher than the intra-cycle $E / I$ indexes $\left[1^{\text {st }}\right.$ test $=1.44 \pm 0.03 ; 2^{\text {nd }}$ test $\left.=1.41 \pm 0.03(P<0,001)\right]$. The cycle-independent $E / I$ indexes from the $1^{\text {st }}$ test were higher than those from the $2^{\text {nd }}$ test $(P=0.04)$. The use of 
the $E$ and I intervals chosen independently of the respiratory cycle where they occurred allowed for the observation of the maximum variation of the parasympathetic activity, denoting the maximal magnitude of the RSA. In addition, the results suggest a reflex accommodation during the $2^{\text {nd }}$ test, making it unnecessary to perform a $2^{\text {nd }}$ test when the $1^{\text {st }}$ one is performed without any technical problem.

Key words: Autonomic nervous system. Respiratory sinus arrhythmia. Autonomic cardiovascular tests.

\section{INTRODUÇÃO}

As atividades simpática e parassimpática constituem, juntamente com a função endócrina, os mecanismos primordiais para a manutenção da homeostasia. Diversas doenças levam a disfunções do sistema nervoso autônomo (SNA), enquanto outras são expressões de tais disfunções.

É sabido que o SNA se adapta ao exercício físico regular. Por exemplo, os atletas de endurance apresentam, por via de regra, menor freqüência cardíaca (FC) quando comparados com indivíduos sedentários ${ }^{1-3}$ e recuperação mais

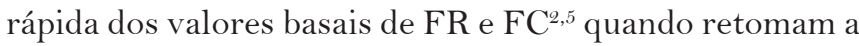
atividade regular após um período sem treinamento. Essas características são conseqüências da adaptação autonômica ao treinamento aeróbico ${ }^{1-5}$. Em alguns raros casos, em que ocorre exacerbação dessa adaptação autonômica, particularmente do aumento da atividade vagal, efeitos adversos na condução do estímulo cardíaco podem aparecer, tornando-se sintomáticos e podendo, inclusive, em casos extremos, indicar a interrupção temporária do treinamento.

Tendo em vista a importância do SNA na gênese, prognóstico e tratamento de diversas síndromes, além de sua notada importância no acompanhamento clínico do atleta, torna-se cada vez mais necessário que o médico, em especial aquele que trabalha com medicina desportiva, conheça o SNA e as formas de avaliá-lo. Um dos testes clássicos utilizados para avaliar o funcionamento do SNA sobre o sistema cardiovascular é chamado arritmia sinusal respiratória (ASR). A ASR é um fenômeno natural, caracterizado pela variação da $\mathrm{FC}$ em função da respiração, sendo primariamente mediada pela modulação parassimpática no nódulo sinusal ${ }^{4-6}$. O teste ASR constitui-se em uma abordagem metodologicamente controlada desse fenômeno. A partir do registro eletrocardiográfico (ECG) identificamse as ondas $\mathrm{R}$ (despolarização ventricular) e medem-se o maior intervalo R-R encontrado durante a expiração (E) e o menor intervalo R-R da inspiração (I). O quociente entre E e I é então utilizado para quantificar a resposta ao tes$\mathrm{te}^{4,7}$.
Apesar de a ASR ser um teste descrito há mais de três décadas ${ }^{5}$, ainda hoje há dúvidas em relação à metodologia mais eficaz para sua realização e correta interpretação. Quando são registrados dois ciclos respiratórios consecutivos, que é o procedimento mais comum, existem dois intervalos R-R chamados de $\mathrm{E}$ (mais longo da expiração) e dois intervalos I (mais curto da inspiração). Qual dos dois intervalos R-R deve ser escolhido para o cálculo do índice E/I? Não existe, na literatura pertinente, uma padronização quanto a essa questão.

O objetivo deste trabalho é comparar os resultados da ASR quando da utilização de critérios diferentes para a seleção dos intervalos R-R do mesmo ciclo ou de ciclos respiratórios diferentes.

\section{MÉTODOS}

Cinqüenta e cinco voluntários [30 homens e 25 mulheres; idade (média \pm desvio padrão) $22 \pm 4$ anos; altura 174 $\pm 9 \mathrm{~cm}$; peso $70 \pm 17 \mathrm{~kg}]$ deram consentimento por escrito para participar do estudo após explicação dos procedimentos a que seriam submetidos. Apenas cinco dos voluntários eram portadores de doenças (dois com asma, um com diabetes melito, um com epilepsia e um com febre reumática); entretanto, os resultados de seus testes não mostraram alterações e, portanto, seus dados foram mantidos na amostra. Todos os indivíduos realizaram dois testes de ASR com intervalo de dois minutos entre eles, sendo cada teste constituído por dois ciclos respiratórios consecutivos, com duração entre 10 e 12 segundos cada um. Os testes foram realizados em decúbito dorsal e cada indivíduo realizou dois ciclos respiratórios variando o volume pulmonar desde a capacidade pulmonar total (inspiração máxima) até o volume residual (expiração máxima). Durante os testes foi obtido traçado contínuo na derivação DII em um ECG de registro termossensível (Funbec ${ }^{\circledR}$, Brasil).

Identificaram-se no traçado de ECG o maior intervalo R-R durante cada expiração (E) e o menor R-R durante cada inspiração (I). Foram então comparados os dois maiores índices $\mathrm{E} / \mathrm{I}$ obtidos das seguintes maneiras: 1) E e I retirados do mesmo ciclo respiratório (intraciclo); 2) E e I retirados de qualquer um dos ciclos respiratórios (cicloindependente).

Os índices E/I obtidos intraciclo e ciclo-independente, em cada um dos testes, foram comparados através da análise de variância (ANOVA) para medidas repetidas, seguidas pelo teste de Bonferroni. Consideramos um valor de $\mathrm{P}$ menor que 0,05 para significância estatística.

\section{RESULTADOS}

Todos os testes foram realizados satisfatoriamente do ponto de vista metodológico. Os índices E/I ciclo-indepen- 


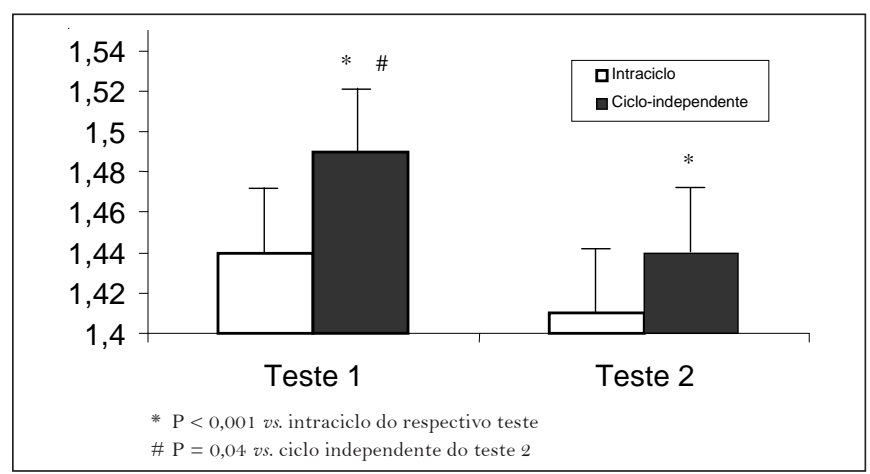

Fig. 1 - Comparação dos índices E/I obtidos intraciclo e ciclo-independente nos dois testes realizados (média \pm erro padrão)

dentes $\left(1^{\circ}\right.$ teste $=1,49 \pm 0,03 ; 2^{\circ}$ teste $\left.=1,44 \pm 0,03\right)$ foram maiores do que os índices $\mathrm{E} / \mathrm{I}$ obtidos intraciclo $\left[1^{\circ}\right.$ teste $=$ $1,44 \pm 0,03 ; 2^{\circ}$ teste $\left.=1,41 \pm 0,03(\mathrm{P}<0,001)\right]$. Os índices $\mathrm{E} / \mathrm{I}$ ciclo-independente do $1^{\circ}$ teste foram maiores do que os do $2^{\circ}$ teste $(\mathrm{P}=0,04)$. Esses resultados estão demonstrados graficamente na figura 1 .

\section{DISCUSSÃO}

O SNA relaciona-se a muitas doenças, seja quanto à sua fisiopatologia, seja no que diz respeito às suas complicações, prognóstico e até mesmo seu tratamento. Neuropatias autonômicas já foram descritas no diabetes mellitus, síndrome da imunodeficiência adquirida, alcoolismo, porfiria, esclerose múltipla e síndrome de Guillain-Barré, entre outras ${ }^{4,8-10}$. Além disso, sabe-se que o desequilíbrio autonômico que ocorre após o infarto agudo do miocárdio ${ }^{11} \mathrm{e}$ na insuficiência cardíaca congestiva ${ }^{12}$ representa um fator de pior prognóstico para esses pacientes. Considerando a vasta área sob controle do SNA e as funções por ele desempenhadas, pode-se imaginar como são diversificados os sinais e sintomas presentes quando, independente da causa, ocorre disfunção de um de seus ramos. Dentre eles podemos citar: hipotensão ortostática, taquicardia, anormalidades da sudorese, alterações da motilidade gastrintestinal, distúrbios esfincterianos e impotência sexual ${ }^{4,8-10}$. Além disso, muitas doenças, como infarto agudo do miocárdio, esofagite de refluxo, íleo paralítico, atonia de bexiga e miastenia grave têm sua gênese associada a distúrbios do funcionamento do SNA.

A atividade autonômica simpática e parassimpática modifica-se agudamente em resposta a estímulos como a respiração e o exercício físico, entre outros. Por outro lado, o treinamento físico gera diversas modificações crônicas no organismo do atleta, incluindo adaptações autonômicas. O aumento da atividade vagal e a redução do tônus adrenérgico que ocorrem em indivíduos treinados aerobicamente levam a menor freqüência cardíaca (FC) durante o repouso e em esforços quando comparada com indivíduos sedentá$\operatorname{rios}^{1-3}$.

O bloqueio atrioventricular do tipo Wenckenbach (tipo I) pode ser considerado um fenômeno comum em atletas bem treinados e provavelmente se relaciona com o maior tônus vagal durante o repouso nestes indivíduos ${ }^{13-15}$. É importante destacar que tal bloqueio desaparece durante o esforço na grande maioria dos casos. Ocasionalmente, tal distúrbio de condução pode exacerbar-se tor nando-se sintomático e, em casos extremos, indicar a interrupção temporária do treinamento ${ }^{15}$.

Vários métodos visando a avaliação, direta ou indireta, do SNA têm sido propostos. As formas diretas são invasivas e inviáveis na prática diária. Em contraste, as formas indiretas são de fácil realização, baseando-se na aplicação de um estímulo quantificável e na observação da resposta a tal intervenção ${ }^{4}$. Mais recentemente, vem sendo utilizada a análise da variabilidade da $\mathrm{FC}$ em repouso sem estimulação específica ou durante as 24 horas do dia. Tal metodologia exige a utilização de um gravador de ECG magnético ou digital e disponibilidade de equipamento informatizado de leitura das gravações munido de programa de análise. Devido a essa razoável sofisticação metodológica, a utilização da análise da variabilidade da FC para avaliação autonômica ainda se limita a poucos centros.

Um método indireto para avaliação da integridade da ação parassimpática sobre o nódulo sinusal é a ASR. Tal método baseia-se no fenômeno de mesmo nome, que consiste na variação da freqüência cardíaca de acordo com a respiração. Esta variação foi descrita pela primeira vez por Stephen Hales em um estudo realizado em cavalos em $1733^{5}$. Desde então, muitos estudos confirmaram a existência desse fenômeno em diversas espécies, inclusive em humanos ${ }^{5-}$ $7,16,17$.

A ASR é um método clássico na investigação de disautonomias, pois se baseia em um fenômeno descrito há mais de dois séculos e, além disso, é facilmente realizado, exigindo apenas um eletrocardiógrafo, um cronômetro e um profissional treinado. Durante a respiração voluntária observa-se sincronia entre taquicardia e inspiração e entre bradicardia e expiração. O padrão da ASR depende da freqüência respiratória e do volume corrente. Entretanto, não existe uma padronização para escolha dos índices E e I utilizados para o cálculo de sua magnitude.

Através da análise dos resultados obtidos percebemos que a utilização de índices E e I escolhidos de forma independente dos ciclos respiratórios realizados consecutivamente, ou seja, não sendo necessariamente retirados de um mesmo ciclo respiratório, permite o registro da maior magnitude do fenômeno. A análise da ASR utilizando tais in- 
tervalos permite, portanto, a quantificação da máxima variação da atividade parassimpática do indivíduo.

A realização de dois testes consecutivos é prática comum quando se realiza a ASR. O objetivo deste procedimento seria, em tese, a utilização do $1^{\circ}$ teste para ambientalização do indivíduo avaliado, obtendo, dessa forma, melhores resultados durante o segundo teste devido à maior facilidade de realização das manobras. Conforme descrito, neste estudo também realizamos dois testes consecutivos. Entretanto, observamos resultados maiores no $1^{\circ}$ teste, apesar de o indivíduo já estar mais familiarizado com a metodologia durante a realização do $2^{\circ}$ teste. Tal resultado sugere uma acomodação do SNA durante o $2^{\circ}$ teste. O fenômeno neurofisiológico de acomodação é descrito para diferentes estímulos ${ }^{18}$, inclusive aqueles relacionados ao sistema cardiovascular ${ }^{19}$. Assim, quando o $1^{\circ}$ teste prosseguir sem intercorrências e o observador considerá-lo metodo-

\section{REFERÊNCIAS}

1. Shin K, Minamitani H, Onishi S, Yamazaki H, Lee M. Autonomic differences between athletes and nonathletes: spectral analysis aproach. Med Sci Sports Exerc 1997;29:1482-90.

2. Dixon EM, Kamath MV, McCartney N, Fallen EL. Neural regulation of heart rate variability in endurance athletes and sedentary controls. Cardiovasc Res 1992;26:713-9.

3. Clausen JP. Effect of physical training on cardiovascular adjustments to exercise in man. Physiol Rev 1977;57:779-815.

4. Castro CL, Nóbrega ACL, Araújo CGS. Testes autonômicos cardiovasculares. Uma revisão crítica. Parte 1. Arq Bras Cardiol 1992;59:75-85.

5. Taha BH, Simon PM, Dempsey JA, Skatrud JB, Iber C. Respiratory sinus arrhythmia in humans: an obligatory role for vagal feedback from the lungs. J Appl Physiol 1995;78:638-45.

6. Grossman P, Karemaker J, Wieling W. Prediction of tonic parasympathetic cardiac control using respiratory sinus arrhythmia: the need for respiratory control. Psychophysiology 1991;28:201-16.

7. Eckberg DL. Human sinus arrhythmia as an index of vagal cardiac outflow. J Appl Physiol 1983;54:961-6.

8. Polinsky RJ, Martin JB. Distúrbios do sistema nervoso autônomo. In: Isselbacher KJ, Braunwald E, Wilson JD, Martin JB, Fauci AS, Kasper DL, editor. Harrison - Medicina interna (tradução), $13^{\text {a }}$ ed, Rio de Janeiro: McGraw-Hill, 1994.

9. Asbury AK. Doenças do sistema nervoso periférico. In: Isselbacher KJ, Braunwald E, Wilson JD, Martin JB, Fauci AS, Kasper DL, editor. Harrison - Medicina interna (tradução), $13^{\text {a }}$ ed, Rio de Janeiro: McGraw-Hill, 1994.

10. Welby SB, Rogerson SJ, Beecching NJ. Autonomic neuropathy is common in human immunodeficiency virus infection. J Infect 1991;23: 123-8. logicamente satisfatório, julgamos que não há necessidade de realização do $2^{\circ}$ teste.

\section{CONCLUSÃO}

Nossos resultados permitem-nos concluir que, em indivíduos jovens, os valores da relação E/I são maiores no $1^{\circ}$ em comparação com um $2^{\circ}$ teste. Em adendo, a maior magnitude da ASR foi observada quando se selecionam os intervalos R-R maior (E) e menor (I) registrados durante qualquer um dos ciclos respiratórios quando se realizam dois consecutivamente. Assim, recomendamos a realização de somente um teste composto de dois ciclos respiratórios consecutivos e a seleção dos intervalos E e I independente do ciclo respiratório onde ocorreram.

\section{AGRADECIMENTOS}

Este projeto tem financiamento da Faperj e do CNPq.

11. La Rovere MT, Bigger JT, Marcus FI, et al: Prognostic value of depressed baroreflex sensitivity: The ATRAMI Study. Circulation 1995; 92(Suppl):I-777.

12. Nolan J, Batin PD, Andrews R, et al. Prospective study of heart rate variability and mortality in chronic heart failure: results of the United Kingdom heart failure evaluation and assessment of risk trial (UKheart). Circulation 1998;98:1510-6.

13. Zeppilli P, Fenici R, Sassara M, Pirrami MM, Caselli G. Wenckebach second-degree A-V block in top-ranking athletes: an old problem revisited. Am Heart J 1980;100:281-94.

14. Kinoshita S, Konishi G. Atrioventricular Wenckebach periodicity in athletes: influence of increased vagal tone on the ocurrence of atypical periods. J Electrocardiol 1987;20:272-9.

15. Zipes DP. Specific arrhytmias: Diagnosis and treatment. In: Braunwald E, editor. Heart disease: a textbook of cardiovascular medicine. 5th ed. Philadelphia: WB Saunders, 1997.

16. Hellman JB, Stacy RW. Variation of respiratory sinus arrhythmia with age. J Appl Physiol 1976;41:734-8.

17. Kim T, Khoo MCK. Estimation of cardiorespiratory transfer under spontaneous breathing conditions: a theoretical study. Am J Physiol (Heart Circ Physiol 42) 1997;273:H1012-2.

18. Rowell LB. Arterial baroreflexes, central commmand, and muscle chemoreflexes: a synthesis. In: Human cardiovascular control. New York: Oxford University Press, 1993.

19. Rowel LB. Reflex control during orthostasis. In: Human cardiovascular control. New York: Oxford University Press, 1993. 